Laboratories, M. Currie Shareholder of: Ironwood Pharmaceuticals, Employee of: Ironwood Pharmaceuticals, H. Schneier Shareholder of: Forest Laboratories, Employee of: Forest Laboratories, J. Johnston Shareholder of: Ironwood Pharmaceuticals, Employee of: Ironwood Pharmaceuticals

\section{PWE-027 MEDIATION ANALYSIS SUPPORTS A DIRECT EFFECT OF LINACLOTIDE (LIN) ON ABDOMINAL PAIN (AP) RELIEF INDEPENDENT OF CONSTIPATION IMPROVEMENT}

doi:10.1136/gutjnl-2013-304907.316

'J MacDougall, ${ }^{2} \mathrm{D}$ Mackinnon, 'B Lavins, ${ }^{3} \mathrm{~A}$ Lembo, ${ }^{4} \mathrm{~S}$ J Shiff, ' $\mathrm{X}$ Hao, ${ }^{1} \mathrm{M}$ Currie, 1."J Johnston. '/ronwood Pharmaceuticals, Cambridge; 'Arizona State University, Tempe; ${ }^{3}$ Beth Israel Deaconess Medical Center, Harvard Medical School, Boston, ${ }^{4}$ Forest Research Institute, Jersey City, United States

Introduction LIN, a 14-amino-acid, minimally-absorbed guanylate cyclase $\mathrm{C}$ agonist (GCCA), significantly improved $\mathrm{AP}$ and complete spontaneous bowel movements (CSBMs) vs placebo (PBO) in 2 Phase 3 IBS-C trials. Recurrent AP in IBS-C may arise from increased visceral hypersensitivity exacerbated by constipation. Relative direct and mediated (increasing CSBMs) LIN effects on AP improvement are unknown. We estimated the direct effect of LIN on improving AP by controlling for a concurrent increase in CSBMs.

Methods Patients (pts) with IBS-C (Rome II criteria) were randomised to LIN $290 \mu \mathrm{g}$ po or PBO for $26 \mathrm{wks}$ in a Phase 3 trial. Pts reported abdominal and bowel symptoms, and rescue medication use daily. Percent improvement from baseline in AP scores was analysed via multilevel mediation analysis to estimate the proportion of the LIN treatment effect attributable to increased CSBMs. CSBMs occurring on the reported AP score day and the previous 6 days were mediation variables. Analysis was performed for Wks 13-26, when LIN and PBO effects on AP were generally constant. An additional analysis summarised AP improvement on a particular day (2-way CSBM stratification: days since last CSBM $[0,1,2, \geq 3$ days $]$ and CSBMs in previous 3 days $[0,1,2, \geq 3$ CSBMs]).

Results Mediation analysis showed the $20 \%$ treatment effect of LIN (48\%) on AP above PBO effect (28\%) resulted from a combined direct effect (18\%) on AP and indirect effect (2\%) mediated by increasing CSBMs. SBMs without a sense of complete evacuation and BMs with rescue medication use did not increase the mediated LIN pain effect. The 2-way CSBM stratification analysis showed improved AP was influenced by time since last CSBM and no. of recent CSBMs (Table). Consistent with a predominant direct LIN effect on AP (per mediation analysis), in each table cell, LIN-treated pts had more AP relief than PBO pts when controlling for CSBM factors.

Percent Improvement in AP Stratified by No. of Recent CSBMs and Time Since Last CSBM by Treatment Group (LIN/PBO [LIN$\mathrm{PBO}$ difference]).

\section{Abstract PWE-027 Table}

\begin{tabular}{lllll}
\hline \multirow{4}{*}{ CSBMs in prior $\mathbf{3}$ days, $\mathbf{n}$} & \multicolumn{4}{l}{ Days since last CSBM, $\mathbf{n}$} \\
\cline { 2 - 5 } & $\mathbf{0}$ (CSBM that day) & $\mathbf{1}$ & $\mathbf{2}$ & $\mathbf{3 +}$ \\
\hline 0 & & & & $45 / 25[20]$ \\
1 & $58 / 43[16]$ & $61 / 37[24]$ & $60 / 40[20]$ & $51 / 38[14]$ \\
2 & $74 / 56[19]$ & $72 / 54[17]$ & $65 / 56[9]$ & $57 / 39[18]$ \\
$3+$ & $76 / 67[9]$ & $76 / 60[16]$ & $76 / 68[8]$ & $74 / 48[25]$ \\
\hline
\end{tabular}

ITT Pop ${ }^{n}$, Wks 13-26, median values (includes pts with multiple values in a particular cell)

Conclusion This analysis supports the hypothesis that LIN has direct effects on AP (over $\mathrm{PBO}$ ), and that AP effects are mediated to a lesser extent by increasing CSBM frequency. Support: Ironwood
Pharmaceuticals Inc \& Forest Laboratories Inc. Editing: CMC funded by Almirall

Disclosure of Interest J. MacDougall Shareholder of: Ironwood Pharmaceuticals, Employee of: Ironwood Pharmaceuticals, D. Mackinnon Consultant for: Ironwood Pharmaceuticals, B. Lavins Shareholder of: Ironwood Pharmaceuticals, Employee of: Ironwood Pharmaceuticals, A. Lembo Consultant for: Ironwood Pharmaceuticals and Forest Laboratories, S. Shiff Shareholder of: Forest Laboratories, Employee of: Forest Laboratories, X. Hao Shareholder of: Ironwood Pharmaceuticals, Employee of: Ironwood Pharmaceuticals, M. Currie Shareholder of: Ironwood Pharmaceuticals, Employee of: Ironwood Pharmaceuticals, J. Johnston Shareholder of: Ironwood Pharmaceuticals, Employee of: Ironwood Pharmaceuticals

\section{PWE-028 SIGNIFICANTLY IMPROVED ABDOMINAL PAIN (AP) WITH LINACLOTIDE (LIN) VS PLACEBO (PBO) IN PATIENTS (PTS) WITH IRRITABLE BOWEL SYNDROME (IBS) REGARDLESS OF BASELINE PAIN SEVERITY IN 2 PHASE 3 TRIALS}

doi:10.1136/gutjnl-2013-304907.317

1."J Johnston, 'W D Chey, 'B Lavins, ${ }^{3} \mathrm{~S} J$ Shiff, 'C Kurtz, 'J MacDougall, ${ }^{3} \mathrm{X}$ Jia, ${ }^{3} \mathrm{H}$ Schneier, ${ }^{1} \mathrm{M}$ Currie. ${ }^{1}$ rronwood Pharmaceuticals, Cambridge; ${ }^{2}$ University of Michigan Heath System, Ann Arbor, ${ }^{3}$ Forest Research Institute, Jersey City, United States

Introduction LIN, a 14-amino-acid, minimally absorbed, guanylate cyclase $\mathrm{C}$ agonist (GCCA), significantly improved abdominal and bowel symptoms in 2 Phase 3 IBS with constipation (IBS-C) trials. Previous IBS trials of other therapies showed inconsistent effects of baseline AP severity on efficacy. This post-hoc analysis determined the LIN effect on AP stratified by pt-reported baseline AP severity.

Methods Pts with IBS-C (Rome II criteria) were randomised to LIN $290 \mu \mathrm{g}$ od po or PBO. Pts rated daily AP at its worst during the previous $24 \mathrm{~h}$ (11-point scale; $0=$ none, $10=$ very severe) during Baseline and Treatment Periods. Using pooled 12-wk ITT data from 2 Phase 3 trials, pts were stratified by mean baseline AP score $(<5$, $\geq 5-<7, \geq 7$ ). At each post-randomisation visit pts rated their relief of AP over the past wk vs baseline (7-point balanced scale; $1=\mathrm{com}$ pletely relieved, $7=$ as bad as I can imagine). The Least Squares (LS) mean change from 2-wk Baseline to 12-wk Treatment Period, \% improvement, difference estimates and $p$-values were calculated (ANCOVA; factors = treatment group, geographic region and study. Covariate $=$ baseline AP)

Results Overall, LIN significantly improved 12 -wk AP vs PBO. LIN also led to significant benefit in all 3 baseline stratified AP subgroups, with pain score decreases of $29-36 \%$ vs $18-20 \%$ for $\mathrm{PBO}$ $(p<0.0001$; Table); improvement was numerically less in pts with milder $(<5)$ baseline severity. Baseline AP scores significantly correlated with absolute magnitude of change from baseline improvement in AP $(r=0.26 ; p<0.0001)$ but not with \% improvement $(r=0.00 ; p=0.9184)$. Pt-rated relief of AP was significantly improved with LIN vs $\mathrm{PBO}$ overall (LS mean 2.9 vs 3.5; $\mathrm{p}<0.0001$ ); relief of AP was also seen for all 3 baseline AP subgroups (baseline AP $<5: 2.8$ vs $3.4 ; \geq 5-<7: 2.9$ vs $3.5 ; \geq 7: 3.0$ vs $3.6 ; p<0.0001$ for all).

\section{Abstract PWE-028 Table 1}

\begin{tabular}{|c|c|c|c|c|c|c|c|c|c|}
\hline \multirow[b]{2}{*}{ Baseline AP group } & \multirow{2}{*}{$\begin{array}{l}\text { Baseline } \\
\text { score }\end{array}$} & \multicolumn{4}{|c|}{ Absolute change (LS mean) } & \multicolumn{4}{|c|}{$\%$ Change } \\
\hline & & LIN & PBO & $\Delta$ & $\mathbf{p}$ & LIN & PBO & $\Delta$ & $\mathbf{p}$ \\
\hline Overall $(n=1602)$ & 5.6 & 1.8 & 1.1 & 0.8 & $<0.0001$ & 32.2 & 18.9 & 13.3 & $<0.0001$ \\
\hline$<5(\mathrm{n}=649)$ & 3.9 & 1.1 & 0.7 & 0.4 & $<0.0001$ & 29.0 & 18.2 & 10.8 & $<0.0001$ \\
\hline$\geq 5-<7(n=577)$ & 5.9 & 2.1 & 1.2 & 1.0 & $<0.0001$ & 35.7 & 19.6 & 16.1 & $<0.0001$ \\
\hline$\geq 7(n=376)$ & 8.0 & 2.6 & 1.5 & 1.0 & $<0.0001$ & 32.1 & 18.7 & 13.3 & $<0.0001$ \\
\hline
\end{tabular}

